\title{
Quasiparticle Interference in Temperature Dependent-STM As a Probe of Superconducting Coherence
}

\author{
Dan Wulin ${ }^{1}$, Yan $\mathrm{He}^{1}$, Chih-Chun Chien ${ }^{1}$, Dirk K. Morr ${ }^{1,2}$ and K. Levin ${ }^{1}$ \\ ${ }^{1}$ James Frank Institute and Department of Physics, University of Chicago, Chicago, Illinois 60637 \\ ${ }^{2}$ Department of Physics, University of Illinois at Chicago, Chicago, Illinois 60607
}

(Dated: November 4, 2018)

\begin{abstract}
In this paper we explore the behavior of the quasi-particle interference pattern (QPI) of scanning tunneling microscopy as a function of temperature, $T$. After insuring a minimal consistency with photoemission, we find that the QPI pattern is profoundly sensitive to quasi-particle coherence and that it manifests two energy gap scales. The nearly dispersionless QPI pattern above $T_{c}$ is consistent with data on moderately underdoped cuprates. To illustrate the important two energy scale physics we present predictions of the QPI-inferred energy gaps as a function of $T$ for future experiments on moderately underdoped cuprates.
\end{abstract}

PACS numbers:

Recently, attention in the field of high temperature superconductivity has turned to characterizing the superconducting phase in the underdoped regime. This phase necessarily differs from that of a conventional $d$-wave superconductor because components of the gap smoothly evolve through $T_{c}$. This leads to a normal state gap or pseudogap above $T_{c}$. Owing to this fact, and unlike a BCS superconductor where the order parameter and the excitation gap are identical, there are very few ways to probe directly something as fundamental as the superconducting order parameter. Within the moderately underdoped samples, which we consider throughout this paper, recent angle resolved photoemission spectroscopy (ARPES) experiments have reported [1, 2] novel signatures of superconducting order. Fermi arcs around the $d$-wave nodes above $T_{c}$ rapidly collapse [2] at the transition to form point nodes. It was argued that, within the superconducting phase, the temperature dependence of the nodal gap has finally provided [1] "a direct and unambiguous observation of a single particle gap tied to the superconducting transition". A complementary and equally valuable probe is scanning tunneling microscopy (STM) and the related quasi-particle interference (QPI) spectroscopy [3, 4, 5, 6]. While this probe, like ARPES, is generally not phase sensitive, a controversy has arisen as to whether these techniques can, as argued experimentally [4, 6], or cannot. as summarized theoretically [5, 7] , distinguish coherent superconducting order from pseudogap behavior.

The objective of this Letter is to provide some resolution to this controversy by studying the temperature evolution of the QPI pattern observed in STM experiments from the superconducting ground state into the pseudogap phase at $T \gtrsim T_{c}$. We employ a microscopically derived preformed pair theory [8] that accounts [9] for all of the complex momentum and temperature dependence of the ARPES spectral gap as described above. The QPI pattern is obtained using the Fourier transform of the local density of states (LDOS) associated with a single impurity. A central result of our study is that, once compatibility with ARPES experiments is incorporated, the observation of the so-called "octet model" QPI [10, 11] (called Bogoliubov QPI or B-QPI) is a direct signature of coherent

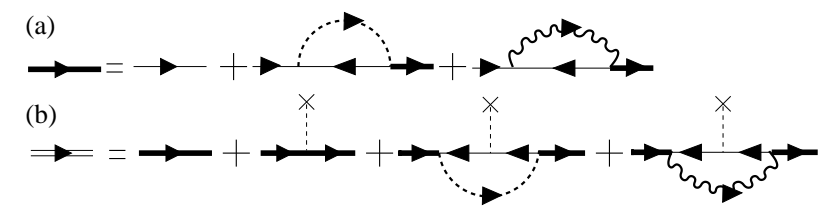

Figure 1: Full Green's function in (a) the clean case (thick solid line), and (b) in the presence of an impurity (double thin lines) to lowest order in $U_{0}$, the impurity strength. A thin line represents the noninteracting normal state Green's function $G_{0}=\left(\omega+\xi_{\mathbf{k}}\right)^{-1}$, and the dotted and wavy lines represent the $t$-matrices $t_{s c}$ and $t_{p g}$ respectively. Panels (a) and (b) are the basis for our ARPES and QPI analysis

superconducting order: sets of consistent octet model vectors can only be found for energies less than the superconducting order parameter energy scale. B-QPI does not persist above $T_{c}$, where the order parameter vanishes.

Our preformed pair theory [8, 9], which has also been successfully applied to the ultracold atoms, is based on BCS-Bose Einstein condensation (BEC) crossover theory, which focuses on a stronger than BCS attractive interaction and the associated short coherence length. The ground state is the usual BCS state with a self consistently determined chemical potential. As outlined elsewhere [8], we solve coupled equations for the fermionic and pair propagators. In the pseudo-gap region below $T^{*}$, there are preformed pairs that become non-condensed pair excitations of the condensate below $T_{c}$. For $T<T_{c}$, both non-condensed and condensed pairs co-exist, but there is a gradual conversion from non-condensed to condensed pairs as temperature is decreased, leading to the conventional BCS ground state as $T \rightarrow 0$.

The pair propagator is related to the $t$-matrix, $t(Q)=$ $t_{s c}(Q)+t_{p g}(Q)$ (with $Q$ defined as a four-vector), where $t_{s c}(Q) \equiv-\frac{\Delta_{s c}^{2}}{T} \delta(Q)$ and $t_{p g}(Q)$ represent the contribution to the $t$-matrix from condensed and non-condensed pairs, respectively. The non-condensed pair contribution $t_{p g}(Q)$ is obtained from a particle-particle $t$-matrix [9] that includes 
one dressed and one bare Green's function [12]. The contribution of the non-condensed pairs to the full self energy $\Sigma(K) \equiv \sum_{Q} t(Q) G_{0}(Q-K) \varphi_{\mathbf{k}-\mathbf{q} / \mathbf{2}}^{2}$ can be well approximated in terms of a pseudogap parameter $\Delta_{p g}(\mathbf{k})$ because $t_{p g}(Q)$ is strongly peaked at small $Q$ for $T$ slightly above $T_{c}$ and $T \leq T_{c}$. Thus, $\Sigma(\mathbf{k}, \omega)$ consists [13] of two terms

$$
\begin{aligned}
\Sigma(\mathbf{k}, \omega) & =\Sigma_{s c}(\mathbf{k}, \omega)+\Sigma_{p g}(\mathbf{k}, \omega) \\
& =\frac{\Delta_{s c}^{2}(\mathbf{k})}{\omega+\xi_{\mathbf{k}}}+\left[\frac{\Delta_{p g}^{2}(\mathbf{k})}{\omega+\xi_{\mathbf{k}}+i \gamma}\right]
\end{aligned}
$$

The two gap parameters, $\Delta_{s c}(\mathbf{k})=\Delta_{s c} \varphi_{\mathbf{k}}$ and $\Delta_{p g}(\mathbf{k})=$ $\Delta_{p g} \varphi_{\mathbf{k}}$, correspond to the superconducting (sc) order parameter and the finite momentum pair gap (pg). The factor $\varphi_{\mathbf{k}}=$ $\left[\cos \left(k_{x}\right)-\cos \left(k_{y}\right)\right] / 2$ ensures d-wave symmetry. The effective gap which appears in the Bogoliubov quasi- particle dispersion is $\Delta(\mathbf{k})=\Delta \varphi_{\mathbf{k}}$ with $\Delta(T) \equiv \sqrt{\Delta_{s c}^{2}(T)+\Delta_{p g}^{2}(T)}$.

The damping $\gamma$ distinguishes the non-condensed pairs from the condensate, as was addressed microscopically in detail in earlier work [14]. We define the ordered phase through the non-vanishing superfluid density, $\rho_{s}$. Microscopic calculations [15] based on Ward identities have established that $\Sigma_{p g}$ effectively cancels in $\rho_{s}$ so that $\rho_{s} \propto \Delta_{s c}^{2}$ as expected.

The Green's function is $G^{-1}(\mathbf{k}, \omega)=\omega-\xi_{\mathbf{k}}-\Sigma(\mathbf{k}, \omega)$ [see Fig. 1 a)]. Here, $\xi_{\mathbf{k}}=-2 t\left(\cos k_{x}+\cos k_{y}\right)-$ $4 t^{\prime} \cos k_{x} \cos k_{y}-\mu$ is the normal state tight binding dispersion with $t=300 \mathrm{meV}, t^{\prime} / t=-0.4$, and $\mu(T=0) / t=$ -1.083 . Here, for definiteness, we take $T_{c}=5.9 \mathrm{meV}$ and $\Delta\left(T_{c}\right)=52 \mathrm{meV}$, with $n=0.85$, representing a moderately underdoped system [16]. We present the simplest approximation [9] of our microscopic theory in order to make our calculations more accessible. To reasonable accuracy [8], $\Delta(T)$ can be found from the BCS gap equation; we approximate [9] $\Delta_{p g}(T)=\Delta(T)\left(T / T_{c}\right)^{3 / 4}$ for $T \leq T_{c}$ and $\Delta_{p g}(T)=\Delta(T)$ for $T>T_{c}$.

The link between ARPES experiments and STM experiments both below and above $T_{c}$ is based on the common spectral function $A(\mathbf{k}, \omega)=-\operatorname{Im} G(\mathbf{k}, \omega) / \pi$. These ARPES experiments constrain the single adjusted parameter $\gamma$ that, nevertheless, has a well understood microscopic origin [14]. We find the presence of a perceptible Fermi arc (with length $\gtrsim 10 \%$ of the Fermi surface) requires [9, 17] that $\gamma\left(T_{c}\right) / \Delta\left(T_{c}\right)>0.2$. We have importantly determined that the behavior of QPI that we report in this Letter is robust for the same regime, $\gamma\left(T_{c}\right) / \Delta\left(T_{c}\right)>0.2$. Specifically, to account for a Fermi arc of about $20 \%$ of the length of the Fermi surface we take $\gamma\left(T_{c}\right) \approx 0.5 \Delta_{p g}\left(T_{c}\right)$. The behavior of the transport lifetime (see Ref. [13] and references therein) suggests that $\gamma$ has a characteristic cubic $T$ dependence below $T_{c}$ and linear above: $\gamma(T)=\gamma\left(T_{c}\right)\left(T / T_{c}\right)^{3}$ for $T \leq T_{c}$, $\gamma(T)=\gamma\left(T_{c}\right)\left(T / T_{c}\right)$ for $T>T_{c}$. Our results are not particularly sensitive to the detailed $T$-dependence, which, for the present purposes, could have been ignored.

In the presence of a non-magnetic impurity, the diagrams contributing to the full Green's function up to first order in

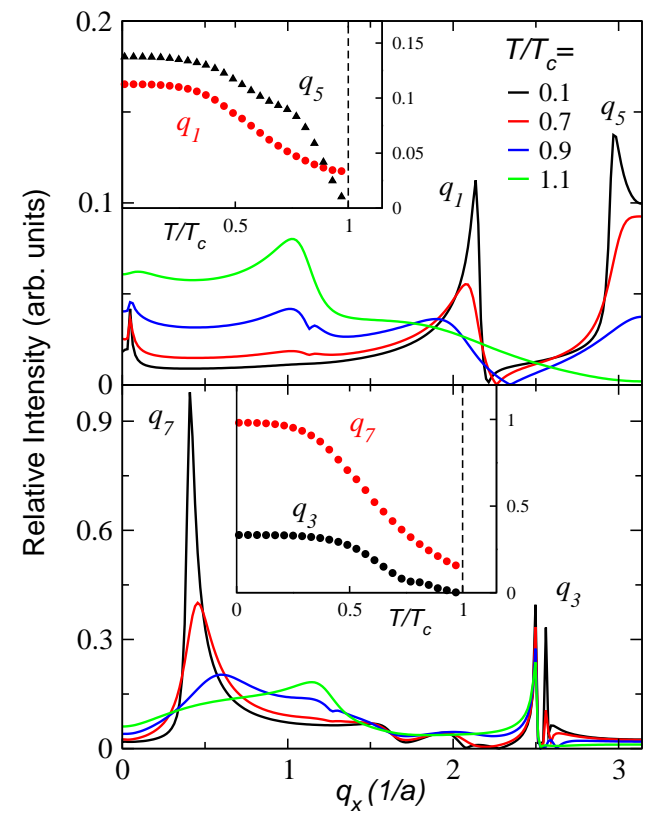

Figure 2: (Color online) One dimensional cuts for fixed frequency $\omega=-10 \mathrm{meV}$ in the (a) horizontal and (b) diagonal directions. Intensities of peaks fall off rapidly as Cooper pairs lose coherence. Insets specify temperature dependence of $q$-vector intensity. All octet peaks disappear at and above $T_{c}$, although there is a finite background for $q_{1}$ and $q_{7}$.

the impurity strength $U_{0}$ are shown in Fig. 11 b). For a single point-like impurity, the Fourier transform of the first order correction to the LDOS is given by

$$
\begin{aligned}
& \delta n(\mathbf{q}, \omega)=-\frac{U_{0}}{\pi} \operatorname{Im}\left[\int \frac{d^{2} k}{(2 \pi)^{2}}(G(\mathbf{k}, \omega) G(\mathbf{k}+\mathbf{q}, \omega)\right. \\
& \left.\left.-F_{s c}(\mathbf{k}, \omega) F_{s c}(\mathbf{k}+\mathbf{q}, \omega)-F_{p g}(\mathbf{k}, \omega) F_{p g}(\mathbf{k}+\mathbf{q}, \omega)\right)\right]
\end{aligned}
$$

where

$$
F_{s c}(\mathbf{K}) \equiv-\frac{\Delta_{s c}(\mathbf{k}) G(\mathbf{K})}{\omega+\xi_{\mathbf{k}}} ; F_{p g}(\mathbf{K}) \equiv-\frac{\Delta_{p g}(\mathbf{k}) G(\mathbf{K})}{\omega+\xi_{\mathbf{k}}+i \gamma}
$$

and $\mathbf{K}=(\mathbf{k}, \omega)$. There are two types of $F F$ terms: the usual one which depends on $F_{s c}$ associated with the sc condensate [11], and a new term (involving $F_{p g}$ ) represented by the last diagram on the right hand side of Fig. 11 b); this reflects the contribution of pre-formed (or non-condensed) pairs. Importantly, this term (which has not appeared in previous work) is required for microscopic consistency.

We have studied the evolution of the QPI pattern with temperature and find that the QPI pattern remains relatively unchanged up to $T=0.5 T_{c}$. This low $T$ behavior is similar to that observed in previous [10] $T=0$ calculations. However, the peak intensity begins to drop rapidly above this temperature. At $T / T_{c}=0.9$ the characteristic (superconducting) QPI pattern becomes smeared out because of the increase in the number of non-condensed pairs. The octet peaks vanish 


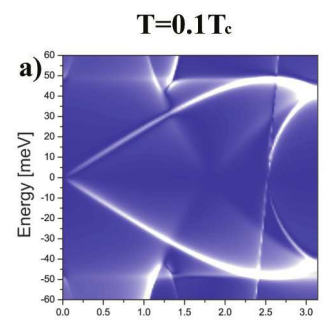

$q_{x}$
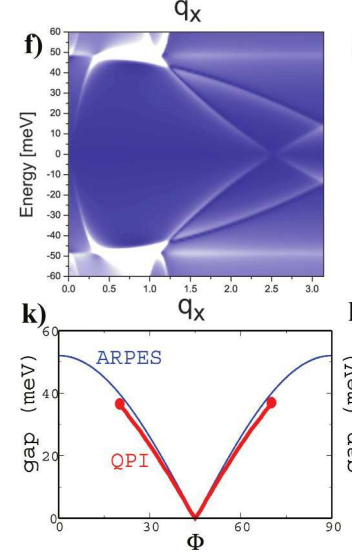
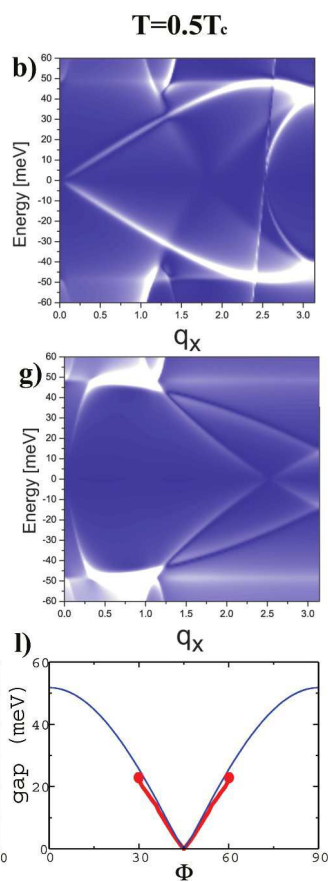

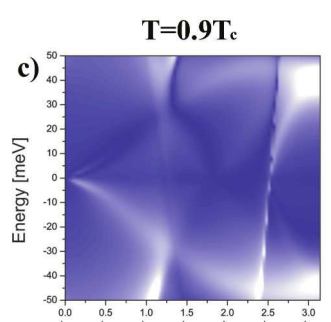

$q_{x}$
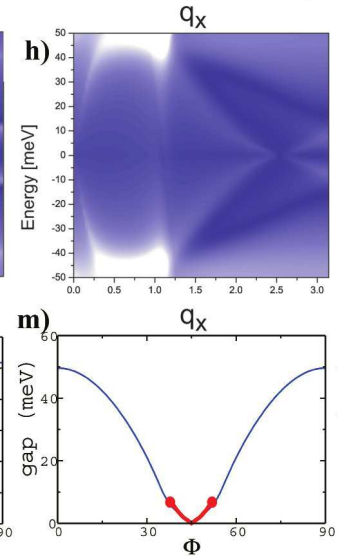

$\mathrm{T}=\mathbf{1 . 1} \mathrm{T}_{\mathrm{c}}$
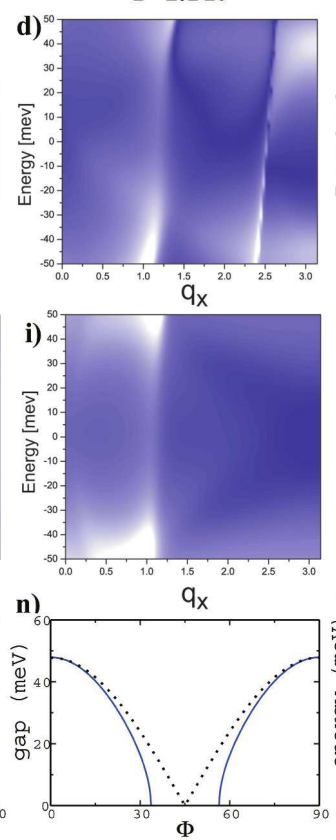
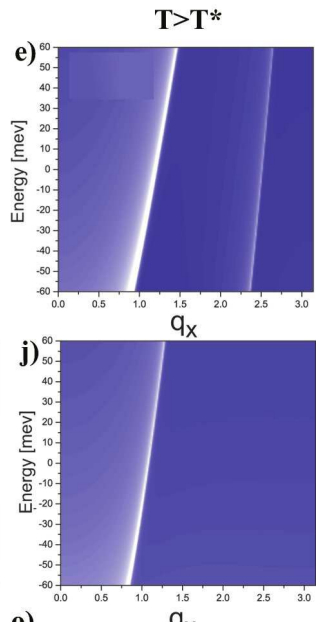

o)

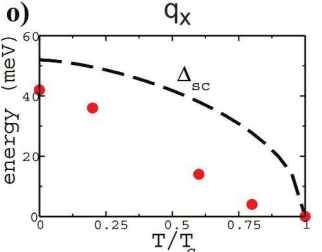

Figure 3: (Color online) First (Second) row shows diagonal (horizontal) cuts along $q_{x}=q_{y}\left(q_{y}=0\right)$ at $\omega=-10$ meV for increasing $T$ from left to right $T / T_{c}=0.1,0.5,0.9$ and 1.1. The last row plots the B-QPI gap (thick red lines) at the same temperatures inferred from pairs of octet vectors along the horizontal and diagonal directions, compared with the ARPES gap (thin blue lines). When the inconsistency between the inferred points on the Fermi surface, $k_{x}$ and $k_{y}$, exceeds $5 \%$ the inversion procedure is terminated. The last figure (3o) on the right shows the temperature dependence of the energy where the octet model breaks down (red dots), which falls with temperature, like the order parameter (black dashed line). The dotted line in the adjacent figure (3n) represents the simple $d$-wave gap shape. It is clear that, as in experiment [1] the ARPES gap varies smoothly across $T_{c}$ as in a second order phase transition.

above $T_{c}$. We find that the QPI pattern above $T_{c}$ is also distinctively different from that of a gapless normal phase [5, 11], as expected in the presence of a pseudogap.

In order to elucidate the temperature dependence of the QPI pattern in more detail, we plot in Fig. [2 $|\delta n(\mathbf{q}, \omega)|$ as a function of $q$ along the horizontal and diagonal directions for $\omega=-10 \mathrm{meV}$. The insets of Fig. 2 (a) and (b) show the temperature dependence of the peak intensity for the dispersing vectors. In agreement with the above discussion, we find that the peak intensities decrease significantly for $T>0.5 T_{c}$. At $T \approx T_{c}$, the peaks associated with the dispersing branches $q_{1}, q_{3}, q_{5}$, and $q_{7}$ all vanish (the peaks associated with $q_{1}$ and $q_{7}$ merge into the background and thus should not be identified as distinctive individual peaks). These insets show that the B-QPI pattern is uniquely associated with superconducting coherence; this strong signature of $T_{c}$ in QPI is correlated with its counterpart in ARPES [1, 2] for moderately underdoped systems.

We turn now to detailed plots of the QPI peak dispersion that reinforce these conclusions. Our main finding is that the dispersive behavior vanishes in the normal state, as is consistent with experiment [6]. This is shown in Figs. 3 (a)-(e) and Figs.3(f)-(j) where $|\delta n(\mathbf{q}, \omega)|$ is plotted as a function of $\omega$ and momentum along the diagonal direction, $q_{x}=q_{y}$, and horizontal direction, $q_{y}=0$. We identify several non-dispersive features in the B-QPI pattern that reflect the normal state. It is not clear to what extent the specifics of the non-dispersive features seen experimentally [6] above $T_{c}$ are present here. In the diagonal cut at $T=0.1 T_{c}$ and at low frequencies and momentum $q \approx 0$, we identify the the octet vector $q_{7}$ [see Fig. 3 (a)], while the peak at momentum $q \approx 2.5$ corresponds to $q_{3}$. In the horizontal cut, we identify the dispersing $q_{1}$ and $q_{5}$-branches, which for $\omega \rightarrow 0$ merge into a single point, $q \approx 2.55$. The momenta $q_{1}, q_{3}, q_{5}$ and $q_{7}$ satisfy the constraints of the octet model for sufficiently low $\omega$.

In the last row of Fig. 3, we probe the effects of superconducting coherence in another way that is strictly confined to the $T \leq T_{c}$ state. Here we display the gap obtained for a given temperature from the B-QPI pattern dispersion shown in the first two rows, using the octet model inversion procedure [4]. Each row was used to generate two sets of points. For definiteness, we take a $5 \%$ consistency level as a necessary condition for the observation of B-QPI. For a given energy $\omega$, the inversion procedure yields points on the Fermi surface, $k_{x}$ and $k_{y}$ such that $\Delta\left(k_{x}, k_{y}\right)=\omega$. The ARPES-derived gap [9] is plotted for comparison. For frequencies $\omega>0.8 \Delta_{s c}$, we find the two sets of Fermi surface points extracted from the octet vectors differ by more than $5 \%$. This leads to a frequency cut-off in the inversion procedure which is plotted in the right-most panel of the bottom row as a function of temperature, where it tracks the superconducting order parameter $\Delta_{s c}$. Consistency between ARPES and B-QPI is found for all 
$T \leq T_{c}$ except in the normal state plot in the last row where the Fermi arc is clearly seen in the ARPES derived spectral gap. One can see from the panel at $T / T_{c}=0.9$ that Fermi arc physics is also apparent below $T_{c}$ in the distortion of the shape of the QPI-inferred gap. Both gaps extrapolate at the antinodes to approximately $50 \mathrm{meV} \approx \Delta(T)$. The segments of $\mathbf{k}$ space where B-QPI inversion is possible correspond to the so-called [4] "Bogoliubov arcs".

While our results for $T \ll T_{c}$ are in agreement with those of earlier studies [10, 11], our results for temperatures above $T_{c}$ differ in several important respects from previous work [5, 7]. In contrast to the pseudogap theory proposed in Ref. [7], we incorporate the presence of Fermi arcs in ARPES above $T_{c}$. This may explain why the pseudogap QPI pattern of Ref. [7] is qualitatively similar to that of the sc state. On the other hand, in Ref. [5] the Fermi arcs were incorporated, but under the presumption that the gap parameters entering the calculation of the QPI pattern and the spectral gaps observed in ARPES experiments were equivalent. The resultant QPI pattern disagreed with the experimental results and it was therefore suggested that the pseudogap phase may be unrelated to precursor superconductivity. In contrast, we argue in our preformed pair scenario that, while the same formalism must be used to address ARPES and STM experiments (as in Fig. 1), the ARPES spectral gap should not be directly employed in the calculations of the QPI pattern.

In summary, in our QPI studies two distinct scales appear, $\Delta_{s c}(\mathbf{k})$ and $\Delta(\mathbf{k})$, which have the same $\mathbf{k}$ dependence. It appears naturally in these STM studies that $\Delta_{s c}(T)$, which vanishes at $T_{c}$, is more influential in the nodal regime, as is widely inferred to be the case [1, 4]. By contrast $\Delta$ is roughly $T$ independent. We extract $\Delta_{s c}(T)$ from the "k-space extinction" of the QPI-inferred gap. (As in Reference [18], we do not find this k-space- extinction point to be directly related to the magnetic zone boundary, as claimed elsewhere [4].) We extract $\Delta$ from the Bogoliubov quasi-particle dispersion, as obtained from the B-QPI inversion procedure. A difference with Reference [4] is that we find the inversion procedure works well even down to the precise nodal point. The failure of this procedure when experimentally implemented near the nodes has not been understood.

An important feature of the present theory is its unique ability to address the ordered phase at general $T$ in the presence of a pseudogap. Other theories are confined to $T \approx 0$ or $T>T_{c}$. Because of this pseudogap it is difficult, but important for theorists to find ways to help identify the very fundamental superconducting order parameter, as we do here. While our microscopically based theory [8] contains one adjusted parameter $\left(\gamma\left(T_{c}\right)\right)$ our theory should not be viewed as principally phenomenological. We have found an important correlation which makes our predictions very robust. The condition on $\gamma\left(T_{c}\right)$ for the appearance of perceptible Fermi arcs in photoemission is essentially the same as the condition for the disappearance of octet peaks in the normal state. In heavily underdoped cuprates (not considered here) we cannot rule out a small contribution of B-QPI in the near vicinity but above $T_{c}$ presumably reflecting the fact that in ARPES the Fermi arcs are not present [18] until substantially above the nominal $T_{c}$. For these more metallurgically complex cuprates near the insulating phase, more detailed experiments and theory are needed to probe the correlations between B-QPI and ARPES.

Note added. After this work was submitted we learned of related STM experiments but on heavily underdoped cuprates by Pushp et al. [19].

This work was supported by Grant Nos. NSF PHY0555325 and NSF-MRSEC DMR-0213745 and by the U.S. Department of Energy under Award No. DE-FG0205ER46225 (D.M.). We thank J.C. Davis, J. Lee and A. Yazdani for helpful discussions.

[1] W. S. Lee, I. M. Vishik, K. Tanaka, D. H. Lu, T. Sasagawa, N. Nagaosa, T. P. Devereaux, Z. Hussain, and Z. X. Shen, Nature 450, 81 (2007).

[2] A. Kanigel, U. Chatterjee, M. Randeria, M. R. Norman, S. Souma, M. Shi, Z. Z. Li, H. Raffy, and J. C. Campuzano, Phys. Rev. Lett. 99, 157001 (2007).

[3] K. K. Gomes, A. Pasupathy, A N Pushp, S. Ono, Y. Ando, and A. Yazdani, Nature 447, 569 (2007).

[4] Y. Kohsaka, C. Taylor, P. Wahl, A. Schmidt, J. Lee, K. Fujita, J. Alldredge, K. McElroy, J. Lee, H. Eisaki, et al., Nature 454, 1072 (2008).

[5] S. Misra, M. Vershinin, P. Phillips, and A. Yazdani, Phys. Rev. B 70, 220503(R) (2004).

[6] M. Vershinin, S. Misra, S. Ono, Y. Abe, A. Yoichi, and A. Yazdani, Science 303, 1995 (2004).

[7] T. Pereg-Barnea and M. Franz, Phys. Rev. B 68, 180506(R) (2003).

[8] Q. J. Chen, J. Stajic, S. N. Tan, and K. Levin, Phys. Rep. 412, 1 (2005).

[9] Q.J.Chen and K.Levin, Phys. Rev. B 78, 020513(R) (2008); C.C.Chien, Y.He, Q.J.Chen, and K.Levin, Phys. Rev. B 79, 214527 (2009).

[10] Q.-H. Wang and D.-H. Lee, Phys. Rev. B 67, 020511 (2003).

[11] A. Capriotti, D. J. Scalapino, and R. D. Sedgewick, Phys. Rev. B 68, 014508 (2003).

[12] L. P. Kadanoff and P. C. Martin, Phys. Rev. 124, 670 (1961).

[13] Q. J. Chen, K. Levin, and I. Kosztin, Phys. Rev. B 63, 184519 (2001).

[14] J. Maly, B. Jankó, and K. Levin, Phys. Rev. B 59, 1354, (1999); J. Maly, B. Jankó, and K. Levin, Physica C 321, 113 (1999).

[15] Q. J. Chen, I. Kosztin, B. Jankó, and K. Levin, Phys. Rev. Lett. 81, 4708 (1998).

[16] A. G. Loeser, Z.-X. Shen, D. S. Dessau, D. S. Marshall, C. H. Park, P. Fournier, and A. Kapitulnik, Science 273, 325 (1996).

[17] A. V. Chubukov, M. R. Norman, A. J. Millis, and E. Abrahams, Phys. Rev. B 76, 180501(R) (2007).

[18] T. Yoshida, M. Hashimoto, S. Ideta, A. Fujimori, K. Tanaka, N. Mannella, Z. Hussain, Z. X. Shen, M. Kubota, K. Ono, et al. (2008), eprint, arxiv: 0812.0155.

[19] A. Pushp, C. V. Parker, A. N. Pasupathy, K. K. Gomes, S. Ono, J. Wen, Z. Xu, G. Gu, and A. Yazdani, Science 324, 1689 (2009). 